Muséologies

Les cahiers d'études supérieures

muséologies

\title{
Utiliser les technologies de l'information et de la communication pour une médiation participative
}

\section{Élisabeth Caillet}

Volume 3, numéro 2, printemps 2009

URI : https://id.erudit.org/iderudit/1033559ar

DOI : https://doi.org/10.7202/1033559ar

Aller au sommaire du numéro

Éditeur(s)

Association Québécoise de Promotion des Recherches Étudiantes en

Muséologie (AQPREM)

ISSN

1718-5181 (imprimé)

1929-7815 (numérique)

Découvrir la revue

Citer cet article

Caillet, É. (2009). Utiliser les technologies de l'information et de la

communication pour une médiation participative. Muséologies, 3(2), 26-35.

https://doi.org/10.7202/1033559ar d'utilisation que vous pouvez consulter en ligne.

https://apropos.erudit.org/fr/usagers/politique-dutilisation/ 
Article un

\section{Utiliser les technologies de l'information et de la}

communication pour une médiation participative

AGRÉGÉE DE PHILOSOPHIE, DOCTEURE EN SCIENCES DE L'ÉDUCATION, EXPERTE EN MÉDIATION CULTURELLE, ÉLISABETH CAILLET A CONÇU ET MIS EN PLACE LES ACTIONS DE MÉdIATION Á LA CITÉ DES SCIENCES ET DE LINDUSTRIE. PUIS ELLE A CONTRIBUÉ Á CRÉER LE DÉPARTEMENT DES PUBLICS DE LA DIRECTION DES MUSÉES DE FRANCE. ELLE A RÉCEMMENT ÉTÉ RESPONSABLE DES EXPOSITIONS ET DE LACTION VERS LES PUBLICS AU MUSÉE DE LHOMME ELLE EST ACTUELLEMENT CHARGÉE DU MUSÉUM VIRTUEL. ELLE A PUBLIÉ A LAPPROCHE DU MUSÉE; LA MÉDIATION CULTURELLE (QUÉBEC: PRESSES DE LUNIVERSITÉ LAVAL, 1995), ACCOMPAGNER LES PUBLICS (PARIS: LHARMATTAN, 2007, COLL. \&PATRIMOINES ET SOCIÉTÉS").

[elisa.callet@gmail.com] 


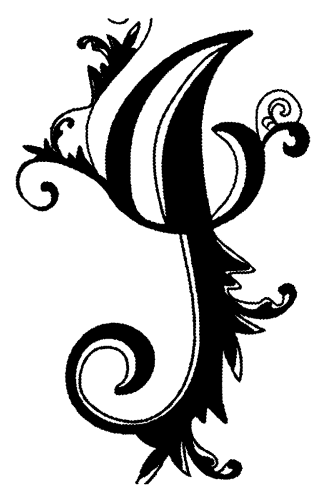

Je présenterai les usages des technologies de linformation et de la communication (TIC) dans le processus de conception de médiation d'une exposition, Naissances, qui s'est tenue au Musée de l'Homme de novembre 2005 à septembre 2006. Je tenterai, à l'aide de cet exemple, de montrer en quoi les TIC facilitent la médiation et permettent une plus grande participation des publics, en les diversifiant et en rendant possible sur la longue durée ce qui n'est généralement possible que dans le cadre limité d'une exposition temporaire. Raison pour laquelle je pense que cette médiation devient réellement participative, objectif que nombre de médiateurs tentent d'atteindre depuis longtemps... sans y parvenir.

Je définis ainsi le principe sur lequel se fonde la médiation participative: "L'attention que nous sommes capables d'apporter à une proposition de formation informelle, comme l'est une exposition, ne peut trouver d'intérêt que si l'on 'intéresse' réellement les destinataires de l'exposition, si l'on parvient à les mettre en situation d'être en quelque sorte 'auteurs' de l'exposition." La médiation participative tente de mettre le public en position d'auteur, de coauteur de ce qu'il perçoit.

Il faut dire d'emblée que le thème de l'exposition, la naissance depuis l'accouchement jusqu'au retour de la mère et de l'enfant dans la société normale, dans différentes cultures, se prêtait particulièrement à une telle tentative, même si les publics visés par la médiation participative n'étaient pas spécifiquement concernés par les TIC puisqu'il s'agissait, d'un côté, de femmes issues de l'immigration et habitant en France et, de l'autre, de professionnels de santé qui ont à prendre part à l'accouchement de ces femmes dans les services hospitaliers. 
La localisation de cette exposition dans le Musée de l'Homme présentait une difficulté: le grand public avait été largement informé de la fermeture des galeries d'expositions ethnographiques qui avaient fait la renommée du Musée del'Homme et dont les collections étaient en cours de transfert vers le futur musée du quai Branly. Tout le monde pensait que le Musée de l'Homme n'existait plus. Il fallait alors manifester la nouvelle de l'existence de ce musée qui prenait alors un autre visage, non encore défini, que l'exposition Naissances préfigurait sans que cela soit véritablement une exposition de préfiguration. Les galeries vides permettaient d'accueillir ainsi des expositions temporaires et laissaient aux activités d'accompagnement la possibilité d'occuper des espaces qui n'étaient pas scénographiés, pas équipés, proches de l'espace d'exposition (ce qui est rarement le cas dans les musées).

Le thème de la naissance nous est vite apparu comme une opportunité permettant à la fois de traiter un sujet qui concernait tout individu et susceptible de symboliser la renaissance du Musée de l'Homme. Enfin, l'utilisation forte des TIC nous conduisait à prendre place dans l'innovation muséale alors que l'image du Musée de l'Homme était liée à une histoire largement dépassée et n’avait pas été mise au standard des scénographies contemporaines.

\section{La démarche}

Lidée de mettre en place un "musée de l'Homme virtuel » à partir de l'exposition Naissances a très vite été acceptée par les commissaires de l'exposition. D'emblée, le travail que nous souhaitions développer pour l'exposition devait être aussi utile pour d'autres expositions à venir. Les solutions techniques que nous allions choisir devaient tenir compte de cette pérennisation qui cherchait à articuler fortement les deux axes du Muséum: la recherche et la diffusion de la recherche.

Par ailleurs, la volonté de faire participer à l'exposition les publics spécifiquement visés (femmes issues de l'immigration, personnels d'institutions professionnelles) n'était pas une idée neuve: elle s'appuyait sur des tentatives anciennes sur lesquelles je ne reviendrai pas ${ }^{[11}$, mais l'usage des TIC pour faciliter l'ensemble de la démarche n'avait encore jamais été tenté. 


\section{S'informer sur l'existant}

Comme pour toute action de médiation, il importait de regarder les actions qui avaient été développées auparavant et de voir ce que nous pouvions leur emprunter pour nos propres activités. Les actions "femmes et musées" initiées par la Direction des musées de France avec les musées Picasso, d'Histoire de Seine Saint-Denis et du Château de Pau avaient montré l'importance qu'il y avait à travailler avec les «femmes-relais ", véritables médiatrices entre les femmes issues de l'immigration et les différentes administrations, pour faire venir ces femmes dans les musées. L'expérience de l'exposition Pauvres de nous que le Musée de l'Homme avait réalisée avec 12 associations (dont en premier Emmaüs alors présidée par Martin Hirsch) avait mis l'accent sur le fait qu'une exposition est capable d'accueillir des productions encadrées par ces associations et réalisées par des personnes extrêmement "désinsérées". Une autre expérience avec le Secours populaire nous avait permis de voir l'efficacité des relais bénévoles pour accueillir un public très important de jeunes enfants non familiers avec un espace muséal. Enfin le travail conduit depuis quelques années par l'Association de promotion et d'insertion par la culture (APIC) nous avait fait rencontrer des professionnels de la relation entre les populations d'autres cultures et les musées parisiens.

Plus encore, nous savions que nous ne pourrions engager le processus de rencontre entre les expôts et les publics éloignés de la culture que par des activités qui les concernent directement, qui entrent dans leurs problèmes: nous avons alors décidé de nous appuyer sur l'expérience du centre de culture scientifique et technique d'île-de-France, Fondation 93, qui avait l'habitude de programmer des rencontres avec des professionnels et des enseignants sur tout sujet relatif aux arts et aux sciences. Nous avons également décidé de nous appuyer sur une exposition réalisée par le Collectif Fusion sur le thème de la diversité de la petite enfance, exposition de photographies prises par les membres de l'association et intitulée Bébés Babel [2].

Conscients enfin de la faiblesse de nos moyens tant sur le plan de l'information que de la diversité de nos collections, nous avons travaillé avec le grand musée du Louvre qui a réalisé, dans ses collections, quatre parcours autour du thème de la naissance.

Ces relations ont progressivement été formalisées par des conventions qui précisaient les apports de chacun : le Musée de l'Homme mettait au service des actions conçues par les associations ses compétences en savoirs scientifiques et en muséographie et accompagnait les différents projets qui se mettaient en place dans chaque lieu de travail des différentes associations. Les associations travaillaient avec leurs usagers habituels en fonction de
[2]

Bébés Babel (2000): à partir de photographies d'albums familiaux et de témoignages recueillis à Sarcelles et à Villiers-le-Bel, mise en parallèle des pratiques traditionnelles de France avec les croyances des populations migrantes liées à la naissance. 
l'évolution du projet d'exposition et des problématiques qu'elles avaient choisi de traiter et des médias de restitution envisagés (un concert, un spectacle, un livre, une exposition de dessins, des affiches, des jeux, une chorégraphie, etc.). Le mode de restitution aux publics du musée, dans un grand espace nommé " Forum » et situé juste à mi-parcours de l'exposition, était précisé collectivement et donnait lieu à une programmation précise : chaque association pouvait occuper le Forum une semaine et laisser trace de ses productions en une accumulation qui permit à cet espace de se remplir progressivement tout au long de la durée de l'exposition (10 mois).

\section{Un groupe de pilotage}

Comme pour la conception d'une exposition, la mise en place de cette médiation participative méritait, par sa nouveauté et ses difficultés propres, le regard de personnes particulièrement vigilantes. C'est ainsi qu'il fut décidé de constituer un groupe de pilotage qui comprenait les professionnels de la médiation les plus aguerris, mais aussi les partenaires administratifs et financiers des associations avec lesquelles nous travaillions. Ce groupe fonctionna pendant les deux années de gestation de l'exposition, avec des formats variables. Y participaient également les représentants des associations partenaires, ce qui permit au groupe de servir de lieu d'écoute, d'échanges et de formation mutuelle.

\section{Relations avec les partenaires}

\section{Partenariat associatif}

Une trentaine d'associations furent ainsi nos partenaires. L'ampleur du projet et la volonté de respecter les expressions que la thématique de l'exposition levait chez les membres des associations nous conduisirent à définir une sorte de déontologie que j'exprimerais ainsi : faire confiance, travailler avec, mais aussi prendre des risques. Ce qui suppose que l'équipe d'animation d'une telle action doit être à la fois chevronnée, présente et certaine de l'appui de son directeur. Il fallait également être en mesure de mettre des moyens à leur disposition : moyens internes (coordination, référents, moyens matériels); moyens externes (démarches conjointes auprès de financeurs réguliers de leurs actions). Il fallait enfin penser à valoriser leurs actions, par une publication collective (mise en ligne sur le site de l'exposition), et à évaluer 
l'ensemble de la démarche, tant pour le musée, qui testait là une possibilité de travail originale que pour les associations, qui outrepassaient souvent ce qu'elles sont censées faire ordinairement ${ }^{[3]}$.

Un partenariat particulier a été mis en place avec l'Association de prévention du site de la Villette (APSV), partenariat directement lié à l'utilisation des TIC dans l'exposition. L'exposition devait être visitée avec un audioguide très perfectionné qui seul permettait d'entendre le son des vidéos et, surtout, complétait les médias visuels de l'exposition par des voix de femmes racontant leur accouchement. Cet audioguide avait été conçu de façon à laisser aux visiteurs la possibilité d'échanger entre eux. Il était géré par une entreprise située à Nice qui en faisait la maintenance à distance. Il était nécessaire de le distribuer à l'entrée de l'exposition et de le récupérer à la sortie. Le personnel des agents de surveillance du musée ne souhaitant pas effectuer cette tâche, l'APSV, par le biais de son entreprise sœur l'AVEJ (Association Villette pour l'insertion des jeunes travaillant particulièrement à l'insertion d'artistes RMIstes), assurait une présence en continu de deux ou trois artistes en formation et en insertion professionnelle; ceux-ci distribuaient les audioguides, en expliquaient le fonctionnement, récupéraient les dispositifs abîmés, informaient le public sur les activités du Forum, répondaient à toute question du public et mettaient à sa disposition le livre d'or.

\section{Partenariat avec les collectivités territoriales La Région île-de-France}

Je n’insisterai pas ici sur ces partenariats absolument nécessaires dans une telle action; ils n'ont rien de spécifique qui soit dû aux TIC. Si ce partenariat particulier a permis la mobilisation des établissements de second degré et donc plus de 300 visites pour des lycéens et des apprentis, il n'a bénéficié des TIC qu'à travers le site Internet dont je décrirai plus loin les éléments. Aucune interactivité particulière n'a été notée, sauf celle induite par une association des femmes de Marseille qui réalisa une série de poèmes qu'elles vinrent lire au Musée de l'Homme à des classes de jeunes élèves du lycée Janson de Saillyy ${ }^{[4]}$. Ces textes ont été écrits lors d'ateliers d'écriture et ont fait l'objet d'une double publication : sur le site de l'exposition et par une maison d'édition ${ }^{[5]}$. Les établissements scolaires avaient demandé que l'exposition soit itinérante, ce que nous pouvions réaliser facilement à partir du site. Malheureusement, une demande mal orientée vers le Service des expositions itinérantes a conduit le Muséum à réaliser une véritable exposition itinérante en dur ${ }^{[6]}$, qui n'avait aucun rapport avec le site Internet que nous avions conçu, ce qui ne permit pas de mener le projet à son terme.
[3]

Une évaluation fut réalisée par Jacqueline Eidelman du Centre d'études et de recherche sur les liens sociaux (CERLIS). [4]

Jumelé avec le Musée de l'Homme. Les jumelages sont des dispositifs établis entre l'Éducation et la Culture afin de réaliser sur la durée des projets en commun.

[5]

Livre publié sous le titre La pré-naissance des âmes, Association Lire Écrire Agir et Secours Populaire de Marseille par les éditions Les Oiseaux de papier (préface de René Frydman), 2007.

[6]

Financée par le ministère des Affaires étrangères, cette exposition circule dans les postes français à l'étranger, mais pas du tout en France. 


\section{Partenariat avec la Ville de Paris}

Ce partenariat a été mis en place dans le cadre de la Politique de la Ville ${ }^{[7]}$ avec les bibliothèques qui ont organisé un espace consacré à la naissance (affiche, catalogue, ouvrages divers) et ont donné à leurs lecteurs un accès Internet au centre de ressources de l'exposition. Un guide de visite et de formation pour les relais jeunes des Antennes jeunes de la Ville (80 antennes) a été conçu et diffusé à la fois sur papier (plaquette) et sur Internet.

Le partenariat avec le Conseil général de Seine-Saint-Denis, par le biais de Fondation 93, a permis d'organiser 21 conférences-débats dans l'espace de débats. Ces activités étaient prioritairement destinées aux organismes de petite enfance du Département, à partir d'une analyse de besoins qui avait été conduite par l'association. Les débats ont été enregistrés et devaient être ensuite accessibles sur le site de l'exposition.

[7]

Cette politique est un outil de travail conjoint entre les collectivités territoriales et les établissements culturels, permettant de privilégier les quartiers en difficulté.

\section{Le Musée de l'Homme, musée virtuel}

Le projet même de musée virtuel prenait son sens dans l'objectif de permettre au musée de rester ouvert pendant les travaux de rénovation. Il cherchait également à élargir les publics et à toucher les jeunes en accroissant l'interactivité (Web 2.0). Il voulait enfin faire connaître la richesse des collections et des travaux des chercheurs (musée laboratoire).

\section{Les rubriques du site et les principales fonctionnalités}

Pour accéder au site : http://www.mnhn.fr/naissances/

Huit onglets étaient accessibles sur la page d'accueil : Accueil, Visites virtuelles, Ressources, Formations, Mon espace, Programmation, Info pratiques, Presse. Nous ne discuterons ici rapidement que de quatre d'entre eux, plus particulièrement importants en termes de musée virtuel.

Tout d'abord, les Visites virtuelles: la principale visite était intitulée Visite adulte. Elle présentait toute l'exposition, salle par salle, selon l'ordre exact de l'exposition, avec tous les objets, tous les textes et toutes les vidéos. Son exhaustivité, qui constituait sa caractéristique, nous paraissait d'autant plus importante que nous savons bien qu'aucun visiteur ne regarde la totalité d'une exposition, aussi longtemps qu'il y reste. 
Une visite plus courte répondait au souhait de disposer d'une « visite enfant "; elle avait été spécifiquement conçue pour le site Internet, alors qu'il n'y en avait pas dans l'exposition matérielle.

La rubrique Ressources permettait d'accéder à tous les éléments de l'exposition, soit à partir de la visite virtuelle, soit par un moteur de recherche: la recherche pouvait se faire par n'importe quel mot clé ou par thème, sousthème, localisation géographique ou type de média. Chaque ressource était indexée avec une quinzaine de métadonnées et une photographie. Un descriptif en texte libre était proposé.

LISTE 1

Liste des métadonnées retenues pour le projet

\begin{tabular}{|c|c|}
\hline $\begin{array}{l}\text { - Référence } \\
\text { - Titre } \\
\text { - Titre d'auteur } \\
\text { - Thème de l'exposition } \\
\text { - Sous-thème de l'exposition } \\
\text { - Genre } \\
\text { - Auteur } \\
\text { - Localisation } \\
\text { - Époque } \\
\text { - Date de réalisation } \\
\text { - Langue } \\
\text { - Dimension } \\
\text { - Résumé } \\
\text { - Niveau (enfants, adultes...) } \\
\text { - Source }\end{array}$ & $\begin{array}{l}\text { - Crédit } \\
\text { - Éditeur / Revue / Producteur } \\
\text { - Année de parution } \\
\text { - Type de média } \\
\text { - Format du média } \\
\text { - Nombre de pages / Durée } \\
\text { - Sous-titrage } \\
\text { - Mots clés } \\
\text { - Statut } \\
\text { - Public restreint } \\
\text { - No d'inventaire } \\
\text { - Date limite d'utilisation } \\
\text { - URL site Web } \\
\text { - URL vidéo ram }\end{array}$ \\
\hline
\end{tabular}

On pouvait enfin trouver dans cette rubrique une bibliographie très complète sur le sujet de l'exposition.

L'onglet Formations proposait diverses utilisations des éléments de l'exposition pour des formations construites par des professionnels ou des intervenants avec lesquels nous avions travaillé: l'une, réalisée par Alain Epelboin, un des commissaires de l'exposition, était une partie d'un cours qu'il avait dispensé à des élèves infirmières et était accessible gratuitement, sur inscription préalable. L'autre avait été réalisée par deux enseignantes d'une formation spécialisée pour les professionnels de santé et traitait de la parentalité.

Cette rubrique était également utilisée pour mettre en ligne les actes du colloque qui s'est tenu au Sénat pendant l'exposition. Ces actes, entièrement enregistrés et disponibles sous forme radiophonique seulement, incluaient une rapide présentation des intervenants. 
La rubrique Forum, probablement mal placée sous la rubrique Formations, était mise à la disposition des partenaires. Elle était utilisée pour proposer une série de productions réalisées par Fabrication Maison, association d'intervenants graphistes qui avaient travaillé pendant plus de deux ans sur le thème de l'exposition avec des enfants et des adultes dans des quartiers "politique de la Ville " "[8] à Paris.

La rubrique Espace personnel offrait à chaque internaute la possibilité de stocker les ressources qu'il désirait conserver pour son usage propre.

Bien évidemment, une évaluation a été faite de cette expérience exceptionnelle. Elle a été confiée au CERLIS qui, sous la direction de Jacqueline Eidelman, a mis en évidence la difficulté qu'il y a à utiliser ce type d'outils quand un musée n'est pas habitué à le faire. On a ainsi pu constater que trop peu de visiteurs ont tenté d'approfondir sur place les données de l'exposition: exposition déjà longue à regarder et qui ne soulevait pas forcément de questions immédiatement formulables.

Nous avons ainsi constaté que la volonté d'un musée de se doter d'un outil en ligne doit être inscrite dans une politique d'ensemble qui ne saurait se réduire à un " coup " ponctuel. Il s'agit ici de créer l'habitude de consulter en ligne des ressources qui prolongent la visite et en transforment les usages. L'intérêt des musées en ligne est donc sans doute plus efficace quand il est possible d'encadrer ces nouveaux usages, comme nous l'avons fait avec l'association partenaire avec laquelle nous avons continué de travailler pendant plus d'un an après la fermeture de l'exposition. Le musée en ligne ne remplace donc en rien la visite d'une exposition, mais en suscite un nouvel usage sur lequel il convient de travailler de façon spécifique. 


\section{Summary}

[Translated by Melissa Rose]

The cultural initiative in museums, which we called "mediation," finds new engaging ways to operate with information technology and communication. Indeed these technologies allow visitors of an exhibition to participate in its animation. Associated with participative initiatives, they strengthen the appropriation of the exhibited contents; they diversify the approaches of a subject and interrelate the learned discourse with that of visitors.

The mediation organized on the occasion Naissances, of an exhibition presented at the Musée de l'Homme in 2005, illustrates that relationship, whose importance seems essential when mediation is applied in a society museum such as the Musée de l'Homme. It attempts to implement two principles of action. From a statement defined by specialists, such as commissioners, curators or researchers, nothing is worth an active method in which the visitor is led to follow the route of those who set up the exposed knowledge. These are inspired by active methods in education and by works on the effectiveness of "real fiction" to popularize the scientific and technical knowledge while preserving their innovative characteristics.

The second principle is based on the fact that, for certain exhibits, visitors may have a way of looking, experiencing, even bringing knowledge to museum objects which is as important as those from specialists who conceived the exhibition and can offer to other visitors comments that are as interesting as those of specialists, particularly because they will be able to criticize them, question them, and thus better understand the concepts emanating from controversies presented by experts.

Interactivity, made possible through new technologies, is thus a new opportunity that museum professionals must seize to bring museums into the XXIst century and anchor them in world debates in which they are inscribed. 